\title{
SUBCUTANEOUS FAT THICKNESS AND THE RISK OF SUPERFICIAL INCISIONAL SURGICAL SITE INFECTION FOLLOWING OPEN APPENDECTOMY
}

\author{
Rai S, Paudel P, Chalise A, Nepal M, Shrestha M
}

Department of Surgery, Nepal Nepal Medical College Teaching Hospital, Attarkhel, Gokarneshwor-8, Kathmandu, Nepal

\begin{abstract}
Obesity is a known risk factor for surgical site infection (SSI). Recent studies have demonstrated that fat burden at the incisional site rather than body mass index (BMI) is a more precise and sensitive measure to predict the risk of SSI. The purpose of the study was to evaluate the correlation between subcutaneous fat thickness (SCFT) at the level of Mc Burney's point and the occurrence of superficial incisional SSI among the patients undergoing open appendectomy. A total of 120 patients who underwent open appendectomy were included in the study. SCFT was measured preoperatively with ultrasonography (USG). The occurrence of superficial incisional SSI was evaluated in relation to SCFT. Previously identified well established risk factors for the development of SSI were also considered by the study. Statistical analysis was performed using SPSS version 17. Overall, superficial incisional SSI was observed in 27 (22.5\%) participants. Mean SCFT at the level of Mc Burney's point among male and female population was $2.26 \mathrm{~cm} \pm 0.74$ (SD) and $2.02 \mathrm{~cm} \pm 0.83$ (SD) respectively. Patients with superficial incisional SSI had a mean fat thickness of $2.80 \mathrm{~cm}$ and those without SSI had mean fat thickness of $1.97 \mathrm{~cm}$. The difference in SCFT was statistically significant $(p=.001)$. More interestingly, the area under the ROC curve was more for SCFT, in comparison to BMI among the patients who developed superficial incisional SSI ( $81 \%$ vs. $73 \%)$. Furthermore, we analysed the occurrence of superficial incisional SSI with SCFT cut off at $2.80 \mathrm{~cm}$, which was statistically significant. As majority of the study population were young adults, risk factors like smoking, diabetes mellitus and nutritional status failed to demonstrate significant correlation with post appendectomy wound site complications. The study demonstrated that the thickness of subcutaneous fat at the site of incision is a good predictor of superficial incisional SSI following open appendectomy and the risk of superficial wound site infection increases as the thickness of subcutaneous fat at the site of incision increases.
\end{abstract}

\section{KEYWORDS}

Appendicitis, incisional, obesity, risk factors, SCFT, SSI

\section{CORRESPONDING AUTHOR}

Dr. Subash Rai,

Registrar,

Department of Surgery,

Nepal Medical College Teaching Hospital,

Attarkhel, Gokarneshwor-8, Kathmandu, Nepal

Email: siriusthulung@gmail.com

Orcid No: https://orcid.org/0000-0002-6448-8370

DOI: https://www.doi.org/10.3126/nmcj.v23i1.36218 


\section{INTRODUCTION}

Acute appendicitis is a common differential among the patients presenting to the emergency department with acute abdominal pain and appendectomy remains one of the most commonly performed emergency procedure. ${ }^{1}$ Despite the existence of various risk reducing pre- and post-operative strategies, surgical site infection (SSI) still is the most common complication after appendectomy, regardless of surgical approach. ${ }^{2}$ SSIs constitute infections of the tissues, organs, or spaces exposed by surgeons during performance of an invasive procedure. ${ }^{3}$ The risk factors for developing SSI are multifactorial and obesity, in particular, has long been validated as a strong risk factor for $\mathrm{SSI}^{4}$. Obesity is associated with multitude of complications including impairment of cutaneous wound healing, total wound failure, and fascial dehiscence. ${ }^{5}$

The most commonly used tool to define obesity in clinical context is body mass index (BMI), a relatively nonspecific assessment of body composition, without any direct measure of adiposity. ${ }^{6}$ Morphometric measures of body composition, in comparison, involves evaluation of certain body parameters such as subcutaneous fat thickness (SCFT), core muscle size, bone mineral density, arterial calcification, and body composition. They can directly quantify the amount of subcutaneous fat at the site of surgical wound. Such targeted measures of body composition may improve assessments for SSI risk, compared with less specific metrics such as BMI. ${ }^{7}$ Morphometric measurements can be obtained by various clinical and radiological modalities. ${ }^{8,9}$ The results of the studies comparing the accuracy and reliability of those modalities in predicting the regional burden of subcutaneous adiposity are largely heterogeneous. ${ }^{10,11}$ We have chosen USG to measure SCFT because of its availability, reliability, and superiority over other modalities. ${ }^{12,13}$

\section{MATERIALS AND METHODS}

This is a hospital based, prospective observational study conducted in Nepal Medical
College Teaching Hospital, Gokarneshwor-8, Kathmandu from 2015 to 2016. Patients between 15 to 60 years, who underwent emergency or elective open appendectomy via various incisions in right iliac fossa were included in the study. SCFT between skin and external oblique aponeurosis, at the level of Mc Burney's point, was measured preoperatively with USG. Other potential risk factors that could contribute to the occurrence of superficial incisional SSI were also taken into account. Open appendectomy performed for complicated appendicitis and those associated with other immunocompromising intraabdominal pathologies were excluded from the study. All the patients were given single dose of preoperative and post-operative intravenous ceftriaxone and metronidazole. Diagnosis of superficial incisional SSI was made as per criteria given by centres for disease control and prevention criteria for defining a SSI, 1999. ${ }^{14}$ Most of the patient were followed up for at least three times, until their thirtieth post-operative day (POD). Data collection was done by using a preformed proforma and the statistical analysis by SPSS version 17.

\section{RESULTS}

A total of 120 patients were included and analysed. The mean age of the study population was 30 years with male and female population of $58 \%$ and $42 \%$ respectively. Majority of the study population included young adults. Seventy eight percentage of patients had normal BMI, as per WHO criteria ${ }^{15}$, and none included in the study had evidence of diabetes mellitus. The total protein level was in the range of 5.50 to $7.50 \mathrm{~g} / \mathrm{dl}$ with mean of $6.8 \mathrm{~g} / \mathrm{dl}$. The difference in SCFT between male and female was statistically insignificant $(p=0.103)$. Patients who were diagnosed with superficial incisional SSI on second POD had mean fat thickness of $2.80 \mathrm{~cm}$ and those without infection had mean fat thickness of $1.97 \mathrm{~cm}$. The difference in SCFT was statistically significant $(\mathrm{p}=0.001)$.

The area under the ROC for SCFT and BMI in patients who had superficial incisional SSI on second POD showed significant difference $(p=0.001)$. The area under the curve was more

Table 1: Mean SCFT and superficial incisional SSI

\begin{tabular}{|lcccc|}
\hline Superficial incisional SSI (second POD) & N & Mean SCFT in cm & Std. Deviation & P value \\
\hline No & 93 & 1.9753 & 0.75537 & \\
Yes & 27 & 2.8000 & 0.52330 & 0.001 \\
\hline
\end{tabular}




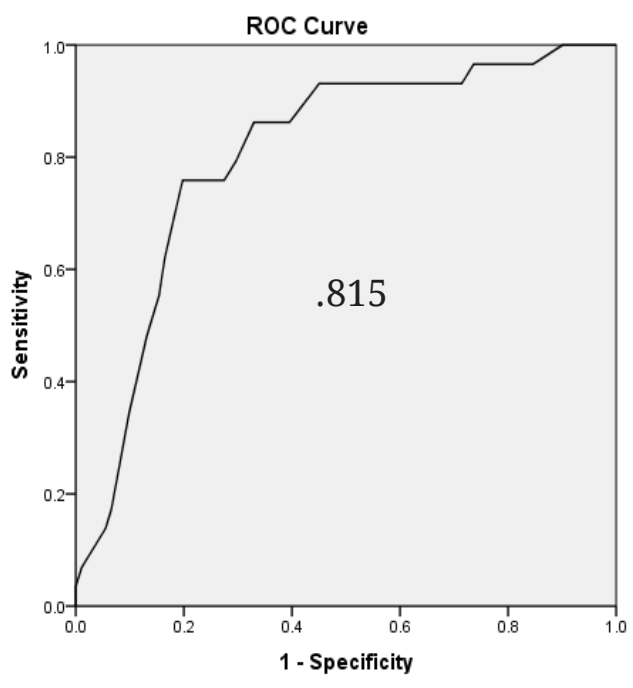

Diagonal segments are produced by ties.

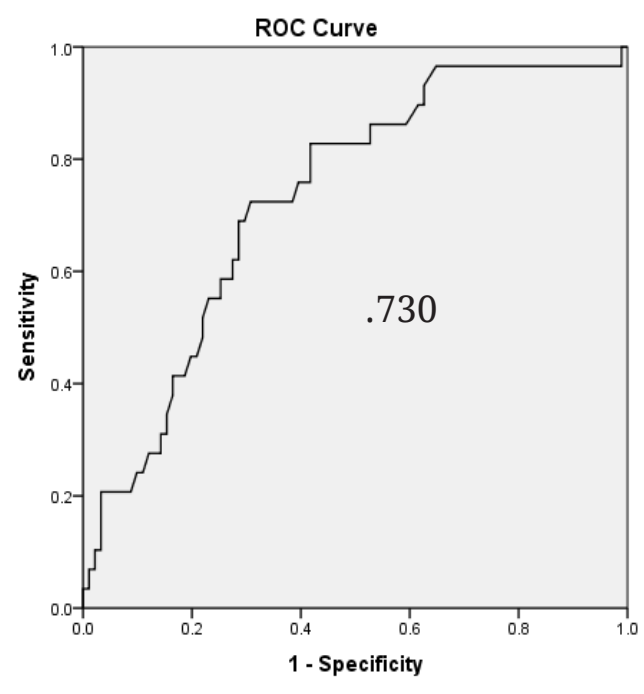

Diagonal segments are produced by ties.

Fig.1: AUC for SCFT and BMI in patients with superficial incisional SSI

Table 2: Occurrence of Superficial Incisional SSI with SCFT cut off at $2.80 \mathrm{~cm}$

\begin{tabular}{|c|c|c|c|c|c|}
\hline & & \multicolumn{2}{|c|}{ SSI, second POD } & \multicolumn{2}{|c|}{ SSI, after seventh POD } \\
\hline \multirow{3}{*}{ SCFT Group } & & No & Yes & No & Yes \\
\hline & $>2.8 \mathrm{~cm}$ & 15 & 15 & 21 & 9 \\
\hline & $<2.8 \mathrm{~cm}$ & 78 & 12 & 87 & 3 \\
\hline$P$ value & & & 0.001 & & 0.001 \\
\hline
\end{tabular}

for SCFT (81\%), when compared to BMI (73\%).

As the area under the ROC curve was more for SCFT, it can be concluded that this variable has more accuracy in predicting the occurrence of superficial incisional SSI than BMI. The area under the curve was maximum, when a cut off fat thickness of $2.80 \mathrm{~cm}$ was assumed. Considering $2.80 \mathrm{~cm}$ as a cut off value of SCFT, the occurrence of superficial SSI was analysed below and above this value, which were statistically significant ( $\mathrm{p}=0.001)$.

The occurrence of superficial SSI was analysed among different BMI groups and the difference was statistically significant $(p=0.049)$. The average duration of surgery was $33.73 \mathrm{~min}$. Both SCFT and BMI showed positive correlation with duration of surgery. The correlation coefficient of SCFT with respect to duration of surgery was more in comparison to BMI ( $\mathrm{R}=0.330$ vs. $\mathrm{R}=0.254$ ).

When SCFT was further categorized, it was observed that the occurrence of superficial incisional SSI increased with the increase in thickness of subcutaneous fat. None of the patients with fat thickness less than $1 \mathrm{~cm}$ had superficial incisional SSI. Out of 26 patients, whose SCFT was more than $2.80 \mathrm{~cm}, 14 \mathrm{had}$ superficial incisional SSI. Furthermore, those with SCFT more than $3 \mathrm{~cm}$ had a 6 fold increase in the odds of developing superficial incisional SSI postoperatively, in comparison to those with SCFT less than $3 \mathrm{~cm}$. Smoking and alcohol consumption did not seem to have significant impact on occurrence of superficial incisional SSI in present study. Similarly, majority of the study population were young adults with normal blood sugar and protein level. These

\section{Table 3: Superficial incisional SSI rates by WHO BMI classification}

\begin{tabular}{|lcc|}
\hline BMI $\left(\mathbf{K g} / \mathbf{m}^{2}\right)$ & WHO obesity class & Superficial incisional SSI \\
\hline$<18.5$ & Underweight & $1 / 2(50 \%)$ \\
$18.5-24.9$ & Normal & $18 / 94(19.14 \%)$ \\
$24.9-29.9$ & Overweight & $10 / 24(41.66 \%)$ \\
\hline
\end{tabular}


factors also failed to demonstrate significant impact upon acute wound outcome.

\section{DISCUSSION}

Our study highlights the fact that obesity indeed has a greater role to play in determining postoperative outcome of patients undergoing elective or emergency surgery. Locally, in a postoperative patient, obesity is a strong risk factor for developing wound site related complications. Systemically, an obese patient is more likely to develop respiratory complications and venous stasis related complications. ${ }^{5,16} \mathrm{~A}$ recent study concluded a greater impact of body fat distribution or habitus in the development of SSI, than overall BMI and obesity did. It is because of the fact that BMI calculation also includes muscle mass and therefore does not always correspond to the amount of fat present. ${ }^{17}$ Our study has demonstrated a similar finding that the distribution of body fat at the surgical site i.e. SCFT is a good clinical measure to predict the risk of postoperative incision site complications and outweighs BMI in reflecting the impact of obesity upon wound outcome.

Beside other clinical relevancies, the true power of measuring regional fat burden lies in the fact that it can provide new insights into the pathophysiology of SSI. Potentially, a surgeon may be able to appreciate the amount of subcutaneous fat on preoperative imaging studies, which could impact intraoperative clinical decision-making and improve the process of informed expressed consent. 18, 19 Although the risk of developing superficial incisional SSI increases with increase in fat burden at the site of incision, there is no corresponding consistent relationship when using BMI, as shown by our study.

When comparing subcutaneous fat and BMI as the only model variables, subcutaneous fat significantly improved model predictions of superficial incisional SSI in the aforementioned study by Lee et al. ${ }^{18}$ Despite having vast differences in the population demographics, the results of our study are comparable to Lee et al. and the predicted variables have shown similar correlation. Yet another study also concluded that SCFT is independently associated and is the most useful predictor of incisional SSI. While BMI was significantly associated with incisional SSI on univariate analysis, this variable lost its significance on multivariate analysis that included SCFT. ${ }^{20}$ It has been established as a significant risk factor for superficial incisional SSI, in a separate study conducted among patients who underwent posterior cervical spine fusion. Patients who developed SSI had a mean thickness of $27 \mathrm{~mm}$ compared with $21 \mathrm{~mm}$ for those who did not $(\mathrm{p}=0.042) .{ }^{17}$

Our study shows that patient with SCFT more than $2.80 \mathrm{~cm}$ measured at Mc Burney's point have a significantly increased risk of developing superficial incisional SSI after open appendectomy, similar to a $3 \mathrm{~cm}$ cut off in a study done among female patients who underwent abdominal hysterectomy. ${ }^{21} \mathrm{~A}$ study done among posterior cervical infusion patients showed 4 fold increase in the odds of developing superficial SSI with a cut off value of SCFT at $50 \mathrm{~mm} .{ }^{16}$ Our study has shown a 6 fold increase in the odds of developing superficial incisional SSI with SCFT more than $3 \mathrm{~cm}$. More importantly, our study depicts that distribution of body mass or actual depth of fat at the surgical site is a greater predictor of superficial incisional SSI than obesity overall. It is obvious that the SCFT measured at midline laparotomy wound and that measured at Mc Burney's point will be different, but the risk opposed to the development of incision related complication remains similar.

In conclusion, subcutaneous fat thickness is a novel morphometric measure of obesity and a good clinical indicator to predict the risk of postoperative superficial incisional SSI among the patients undergoing open appendectomy. The risk of superficial wound site infection increases as the thickness of subcutaneous fat at the site of incision increases.

\section{REFERENCES}

1. Andersson R. Meta-analysis of the clinical and laboratory diagnosis of appendicitis. Br J Surg 2004; 91: 28-37.

2. Xiao Y, Shi G, Zhang J et al. Surgical site infection after laparoscopic and open appendectomy: A multicenter large consecutive cohort study. Surg Endosc 2014; 29: 1384-93.
3. Schwartz S, Brunicardi F, Andersen D. Schwartz's Manual of Surgery. 8th ed. New York: McGrawHill, Medical Pub. Division; 2006.

4. Hourigan J. Impact of obesity on surgical site infection in colon and rectal surgery. Clin Colon Rectal Surg 2011; 24: 283-90. 
5. Pierpont Y, Dinh T, Salas R et al. Obesity and surgical wound healing: A current review. ISRN Obes 2014; 2014: 1-13.

6. Vanderwall C, Randall Clark R, Eickhoff J, Carrel A. BMI is a poor predictor of adiposity in young overweight and obese children. BMC Pediatr 2017; 17: 135.

7. Levi B, Zhang P, Lisiecki $J$ et al. Use of morphometric assessment of body composition to quantify risk of surgical-site infection in patients undergoing component separation ventral hernia repair. Plast Reconstr Surg 2014; 133: e559-66.

8. Duren D, Sherwood R, Czerwinski et al. Body composition methods: Comparisons and interpretation. J Diabetes Sc Technol 2008; 2: 1139-46.

9. Ellis K. Human body composition: In vivo methods. Phys Rev 2000; 80: 649-80.

10. Browning L, Mugridge O, Chatfield $\mathrm{M}$ et al. Validity of a new abdominal bioelectrical impedance device to measure abdominal and visceral fat: Comparison with MRI. Obesity 2010; 18: 2385-91.

11. Johnson KE, Naccarato IA, Corder MA, Repovich WES. Validation of three body composition techniques with a comparison of ultrasound abdominal fat depths against an octopolar bioelectrical impedance device. Int'l J Exerc Sci [Internet] 2012; 5: 205-13. Available from: https://europepmc.org/articles/PMC4853002

12. De Lucia Rolfe E, Sleigh A, Finucane FM et al. Ultrasound measurements of visceral and subcutaneous abdominal thickness to predict abdominal adiposity among older men and women. Obesity 2009; 18: 625-31.
13. Wagner DR. Ultrasound as a tool to assess body fat. J Obes 2013; 2013: 1-9.

14. Horan TC, Gaynes RP, Martone WJ, Jarvis WR, Grace Emori T. CDC definitions of nosocomial surgical site infections, 1992: A modification of CDC definitions of surgical wound infections. Am J Infect Control 1992; 20: 271-4.

15. Samuelson G. Physical Status: The Use and Interpretation of Anthropometry. WHO Technical Report Series. Acta Paediatr 1997; 86: 280.

16. Lee JJ, Odeh KI, Holcombe SA et al. Fat thickness as a risk factor for infection in lumbar spine surgery. Orthopedics 2016; 39: e1124-8.

17. Mehta AI, Babu R, Sharma R et al. Thickness of subcutaneous fat as a risk factor for infection in cervical spine fusion surgery. J Bone Joint Surg Am 2013; 95: 323-8.

18. Lee JS, Terjimanian MN, Tishberg LM et al. Surgical site infection and analytic morphometric assessment of body composition in patients undergoing midline laparotomy. $J$ Am Coll Surg 2011; 213: 236-44.

19. Tjeertes E, Hoeks S, Beks S et al. Obesity - a risk factor for postoperative complications in general surgery? BMC Anesthesiol 2015; 15: 112.

20. Fujii T, Tsutsumi S, Matsumoto A et al. Thickness of subcutaneous fat as a strong risk factor for wound infections in elective colorectal surgery: Impact of prediction using preoperative CT. Dig Surg 2010; 27: 331-5.

21. Soper D, Bump R, Hurt W. Wound infection after abdominal hysterectomy: Effect of the depth of subcutaneous tissue. Am J Obstet Gynecol 1995. p. 465-71. 